OPEN ACCESS

Edited by:

Sandra G. Velleman, The Ohio State University, United States

Reviewed by:

Walter Gay Bottje, University of Arkansas, United States

Michael Babak Papah, University of Pennsylvania, United States

Francesca Soglia,

University of Bologna, Italy

*Correspondence:

Tomohito Iwasak

iwasaki@rakuno.ac.jp

Takafumi Watanabe

t-watanabe@rakuno.ac.jp

Specialty section: This article was submitted to Avian Physiology, a section of the journal

Frontiers in Physiology

Received: 29 March 2020 Accepted: 11 May 2020

Published: 16 June 2020

Citation:

Hosotani M, Kawasaki T,

Hasegawa Y, Wakasa Y, Hoshino M,

Takahashi N, Ueda H, Takaya T,

Iwasaki T and Watanabe T (2020)

Physiological and Pathological Mitochondrial Clearance Is Related

to Pectoralis Major Muscle

Pathogenesis in Broilers With

Wooden Breast Syndrome.

Front. Physiol. 11:579.

doi: 10.3389/fphys.2020.00579

\section{Physiological and Pathological} Mitochondrial Clearance Is Related to Pectoralis Major Muscle Pathogenesis in Broilers With Wooden Breast Syndrome

\author{
Marina Hosotani', Takeshi Kawasaki², Yasuhiro Hasegawaㄹ, Yui Wakasa', \\ Maki Hoshino ${ }^{1}$, Naoki Takahashi' ${ }^{1}$ Hiromi Ueda ${ }^{1}$, Tomohide Takaya ${ }^{4}$, Tomohito Iwasaki ${ }^{*}$ \\ and Takafumi Watanabe ${ }^{1 *}$ \\ ${ }^{1}$ Department of Veterinary Anatomy, School of Veterinary Medicine, Rakuno Gakuen University, Ebetsu, Japan, ${ }^{2}$ Research \\ Office Concerning the Health of Humans and Birds, Abashiri, Japan, ${ }^{3}$ Department of Food Science and Human Wellness, \\ College of Agriculture, Food and Environment Science, Rakuno Gakuen University, Ebetsu, Japan, ${ }^{4}$ Department \\ of Agricultural and Life Science, Faculty of Agriculture, Shinshu University, Nagano, Japan
}

Wooden breast syndrome (WB) constitutes an emerging myopathy in the pectoralis major muscle (PM) of broiler chickens, characterized by myofiber hypertrophy and degeneration along with severe fibrosis. WB pathogenesis has been considered to involve hypoxia induced by rapid growth of the PM. In this study, we focused on mitochondrial morphology and dynamics in the myofibers, as these organelles are sensitive to damage by hypoxia, and examined the effects on WB pathogenesis. Specifically, the PMs of a flock of 35 broilers at 50 days of age were evaluated. First, the severity of disease in each bird was determined by measuring histopathological indices including the fibrotic area (FA) in the muscle and circularity of myofibers (CM). These values were $29.4 \pm 9.6 \%$ and $0.70 \pm 0.042$, respectively, showing variety among the flock. Myofiber vacuolization was observed in all birds including numerous small- or large-rimmed vacuoles, with the former consisting of ultrastructurally autophagosome-like vacuoles engulfing degenerated mitochondria. The large-rimmed vacuoles frequently occurred in the PMs with more severe FA and $\mathrm{CM}$, indicating a relationship between altered autophagy/mitophagy and WB severity. Next, the expression levels of hypoxia-adaptive and mitochondrial dynamics-related genes were analyzed, and their correlations with the histopathological indices were examined. The histopathological indices were negatively correlated with the expression of vascular endothelial growth factor A (VEGFA), indicating that less angiogenesis owing to weakened hypoxia-inducible factor signaling induces more severe WB pathology. In addition, the observed negative correlation with mitochondrial dynamics-related genes implied that WB pathology deteriorates concomitant with reduced mitochondrial dynamics. Furthermore, the expression of mitochondrial dynamics-related genes showed strong positive correlation with that of VEGFA and autophagy-/mitophagyrelated genes. These results revealed that the PMs of broilers possess the mechanism 
of physiological clearance of mitochondria damaged by the hypoxia resulting from the continuous mitochondrial dynamics and autophagy/mitophagy accompanying rapid PM growth. In turn, the altered mitochondrial clearance induced by chronic hypoxia and the accumulation of damaged mitochondria likely underly the severe pathological features of WB.

Keywords: wooden breast syndrome, pathology, hypoxia, mitochondria, autophagy, mitophagy

\section{INTRODUCTION}

In poultry production, meat quality impairment following the selective breeding of fast-growing broiler chickens necessary for supplying the large global demands of chicken meat represents an emerging problem worldwide (Kuttappan et al., 2013; Petracci et al., 2015). Specifically, genetic selection strategies have aimed to increase the yield of the pectoralis major muscle (PM), which constitutes the most superficial breast muscle (Bailey et al., 2015). However, the PMs of some broilers exhibit severe hardness, pale color, and small hemorrhages upon palpation and macroscopic observation in the poultry slaughterhouse (Sihvo et al., 2014) and ultimately cannot be utilized, which produces large economic losses (Abasht et al., 2016). This emerging myopathy, termed Wooden breast syndrome (WB) (Petracci et al., 2019), are characterized by histopathological features such as hypertrophy, necrosis and regeneration of myofibers, macrophage infiltration, and fibrosis (Sihvo et al., 2014; Clark and Velleman, 2016; Kawasaki et al., 2018). Consequently, the achievement of both high production efficiency of PM yield and reduction in the WB presentation is a highly desirable goal in the poultry industry.

In particular, broilers with the same genetics can show either no, mild, or severe WB symptoms at the same age even if raised under identical circumstances (Papah et al., 2018). WB severity is more highly associated with muscle development than with the overall growth of the body (Griffin et al., 2018). WBaffected PMs start to deteriorate after approximately 2 or 3 weeks (Radaelli et al., 2017; Kawasaki et al., 2018), such that a flock of broilers at later life would include birds at both initial and chronic stages of WB.

Wooden breast syndrome pathogenesis is considered to derive from insufficient angiogenesis during the rapid growth of the PMs leading to the production of oxidative stress and hypoxia (i.e., reduced oxygen availability) in the PMs, resulting in their myopathies (Mutryn et al., 2015; Malila et al., 2019). Hypoxia-inducible factor 1 (HIF1), which constitutes the primary transcriptional regulator produced in response to hypoxia in both physiological and pathological contexts, can increase vascularization and maintain oxygen homeostasis (Ziello et al., 2007; Thomas and Ashcroft, 2019). Specifically, HIF1-mediated cellular adaptations under hypoxic conditions regulate the metabolism, morphology, mass, and distribution of mitochondria through their fusion, fission, and autophagy/mitophagy (Thomas and Ashcroft, 2019). Notably, it has been reported that the expression of HIF1 transcripts and related genes is upregulated in the PMs of broilers with both moderate and severe WB compared to that of unaffected birds (Malila et al., 2019), with the first molecular signs of WB development being observed from 2 weeks of age (Papah et al., 2018). Nevertheless, different birds may exhibit individual susceptibilities to the development of such defects, as some are likely able to better counteract the development of hypoxic conditions giving rise to the cascade of events responsible for symptom development. As oxygen is essential for mitochondrial metabolism, we thus hypothesized that the damage to mitochondria mediated by hypoxia serves as the first step in WB pathogenesis, followed by muscle pathology such as hypertrophy and fibrosis. To evaluate the effect of mitochondrial damage on WB pathogenesis, in this study, we therefore performed PM histopathology and mitochondrial morphology assessments and analyzed the expression levels of mitochondrial dynamics-related genes in a flock of broilers including those with moderate to severe WB.

\section{MATERIALS AND METHODS}

\section{Animals and Specimens}

Animal experimentation was approved by the Rakuno Gakuen University Institutional Animal Care and Use Committee (No. VH18A6) in accordance with the Act on Welfare and Management of Animals of the Japanese government. A total of 36 male chicks (Ross308) hatched in the university farm were raised until day 50 . All birds were observed daily by animal stock technicians, and clinical conditions were checked by a poultry veterinarian as necessary during the rearing period. The birds were weighed and determined as clinically unaffected or exhibiting signs of WB by palpating the breast and via the simple method of wing lift examination, which can be performed in live birds by gently lifting up the wings of each bird to assess the ability to achieve back-to-back wing contact (Kawasaki et al., 2016) at 15, 44, and 50 days of age (just prior to euthanasia). The birds were housed in clean concrete-floored pen $(1.8 \times 1.2 \mathrm{~m})$ covered with clean soft sawdust litter under suitable air conditioning and hygiene management and had free access to commercial corn-based diet and water. At 50 days of age, 35 birds weighing over $3,300 \mathrm{~g}$ were selected. The body weights of selected birds were $4,090 \pm 478 \mathrm{~g}$. All birds were euthanized by exsanguination under deep anesthesia induced using 20-30 $\mathrm{mg} / \mathrm{kg}$ pentobarbital sodium into the radial vein with sterilized disposable $2.5-\mathrm{ml}$ syringes and 23 -gauge needles. The muscle samples were collected from one-third of the anterior part of the PM where fibrosis frequently occurs (Clark and Velleman, 2016; Hasegawa et al., 2020), fixed with Bouin's fixative (Polysciences, Inc., Warrington, PA, United States) at $4^{\circ} \mathrm{C}$ for 
histological analysis, fixed with half-Karnovsky's fixative [2.5\% glutaraldehyde, $2 \%$ paraformaldehyde, $0.1 \mathrm{M}$ cacodylate buffer (CB), $\mathrm{pH} 7.4]$ at $4^{\circ} \mathrm{C}$ for ultrastructural analysis, or immersed in RNAlater (Qiagen, Hilden, Germany) at $4^{\circ} \mathrm{C}$ for the analysis of gene expression. The PMs fixed with Bouin's fixative were embedded in paraffin and cut into $4-\mu \mathrm{m}$-thick sections, which were then used for hematoxylin-eosin (HE) and Azan staining.

\section{Histoplanimetry of the PM}

To evaluate the indices of muscle pathology, fibrotic area (FA) in the muscle and circularity of myofibers (CM) were measured. Several previous studies have reported that the primary histopathological characteristics in WB include collagen deposition (fibrosis) in the interstitium and lack of polygonality of myofibers (Sihvo et al., 2014; Kawasaki et al., 2018). Therefore, we chose the FA and $\mathrm{CM}$ as representative histopathological parameters to show the degree of WB-mediated deterioration. To calculate the FA, the area of myofibers $(a)$ and the whole area of muscle $(b)$ in four different randomly selected regions of individual Azan-stained histological sections of each specimen were measured using Image Pro software V10.0.04 (Media Cybernetics, Inc., Rockville, MD, United States). The FA was calculated as follows: FA $(\%)=100 \times$ [whole area of the muscle $(b)$ - area of myofibers $(a)] /$ whole area of the muscle $(b)$. According to the FA value, the birds were divided into stages IVI by groups of $5 \%$ units; i.e., stage I $(<20 \%, n=6)$, stage II $(20-25 \%, n=5)$, stage III $(25-30 \%, n=6)$, stage IV $(30-35 \%$, $n=5)$, stage $\mathrm{V}(35-40 \%, n=9)$, and stage VI $(>40 \%, n=4)$. To calculate the CM, 200 myofibers on four different randomly selected regions of individual HE-stained histological sections of each specimen were manually traced, and their CMs were calculated using ImageJ ver1.52a (National Institutes of Health, Bethesda, MD, United States). The formula for CM calculation is $4 p \times$ area of myofiber/(perimeter of myofiber $)^{2}$.

\section{Ultrastructural Analysis}

For transmission electron microscope (TEM) analysis, the PMs of sample numbers $1,2,6,9,10,15,17,19$, and $33(n=9)$ fixed by half-Karnovsky's fixative were used for specimen preparation. The contrast of the mitochondrial membrane structures was enhanced for TEM by heavy metal block staining, as described previously (Thai et al., 2016). Briefly, the fixed PMs were washed four times with a solution containing $0.1 \mathrm{M} \mathrm{CB}(\mathrm{pH}$ 7.4) and $2 \% \mathrm{OsO}_{4}$ (TAAB Laboratories Equipment Ltd., Berks, United Kingdom) in $0.15 \% \mathrm{~K}_{4}(\mathrm{CN})_{6}$ (Nacalai Tesque, Inc., Kyoto, Japan) for $4 \mathrm{~min}$ each at $4^{\circ} \mathrm{C}$ and soaked in the same solution for $1 \mathrm{~h}$. After four washes with distilled water (4 min each), the samples were immersed in $0.1 \%$ thiocarbohydrazide (Sigma Aldrich Co. LLC., St. Louis, MO, United States) for $20 \mathrm{~min}$ at room temperature, then washed again with distilled water and immersed in $2 \% \mathrm{OsO}_{4}$ for $30 \mathrm{~min}$ at room temperature. Following further distilled water washing, the samples were immersed in $1 \%$ uranyl acetate at $4^{\circ} \mathrm{C}$ overnight, washed again in distilled water, and immersed in Walton's lead aspartate solution at $60^{\circ} \mathrm{C}$ for $30 \mathrm{~min}$. Finally, the samples were dehydrated through an ethanol series, transferred to QY-1, and embedded in epoxy resin (Quetol 812; Nissin EM Co. Ltd., Tokyo, Japan). The tissue block was sliced into 80 -nm-thick sections perpendicular to the long axis of the myofiber using an ultramicrotome (JUM-7; JEOL Ltd., Tokyo, Japan). The sections were collected on a 100-mesh copper grid, and the structures were observed by TEM (HT-7700; Hitachi High Technology Co., Tokyo, Japan) at an acceleration voltage of $80 \mathrm{kV}$.

\section{Reverse Transcription and Quantitative Real-Time Polymerase Chain Reaction}

The PMs of broilers were homogenized using a BioMasher (Nippi, Inc., Tokyo, Japan), and total RNA from samples was purified using TRIzol reagent (Life Technologies, Carlsbad, CA, United States) according to the manufacturer's instructions. The purified total RNA was used as a template to synthesize complementary DNA (cDNA) using ReverTra Ace qPCR RT Master Mix (Toyobo Co., Ltd., Osaka, Japan). Quantitative realtime PCR (qPCR) analysis was performed on the cDNA using THUNDERBIRD SYBR qPCR Mix (Toyobo Co., Ltd.) and genespecific primers (Table 1, Sigma-Aldrich). The qPCR cycling conditions were $95^{\circ} \mathrm{C}$ for $1 \mathrm{~min}$, followed by 40 cycles of $95^{\circ} \mathrm{C}$ for $15 \mathrm{~s}$, and $60^{\circ} \mathrm{C}$ for $45 \mathrm{~s}$. Data were normalized against the expression level of actin, beta $(A C T B)$ and analyzed using the $\Delta \mathrm{Ct}$ method for correlation analysis and the $\Delta \Delta \mathrm{Ct}$ method for stage-related comparison of gene expression.

\section{Statistical Analysis}

Results were expressed as the mean \pm standard error (SE). Data among three or more groups were compared using the Tukey test $(P<0.05)$. Data between two groups were compared using the Student's $t$ test $(P<0.05)$. Correlations between two parameters were analyzed using Spearman's correlation test $(P<0.05)$.

\section{RESULTS}

\section{Histopathological Observation of the PM}

As shown in Figure 1, the histological sections of the PMs stained with Azan and HE showed both mild and severe fibrotic pathology, as revealed by the collagen distribution stained blue with Azan, and myofiber hypertrophy. The values of FA and CM in the PMs of each broiler are shown in Table 2; mean values in all birds were $29.4 \pm 9.6 \%$ and $0.70 \pm 0.042 \%$, respectively. According to FA, the birds were grouped into six stages. The CM tended to increase with higher stage (Table 2) and significantly positively correlated with the FA [Spearman's correlation coefficient $(\rho)=0.6246, P<0.0001]$. In PMs with relatively low levels of FA and $\mathrm{CM}$ among all broilers (stage I), not only the normal polygonal myofibers but also myofibers with numerous small vacuoles were observed (Figures 1A-C). In comparison, in PMs with relatively high levels of FA and CM (stage VI), several types of myofibers with pathology could be seen: myofibers with numerous small vacuoles and large-rimmed vacuoles, degenerated myofibers, myofibers with small caliber, and split fibers (Figures 1D,E). During the observation period, we obtained clinical information including body weight at 15, 44, and 50 days, weight gain ratio from 15 to 50 days, and results 
TABLE 1 | List of primers used in the study.

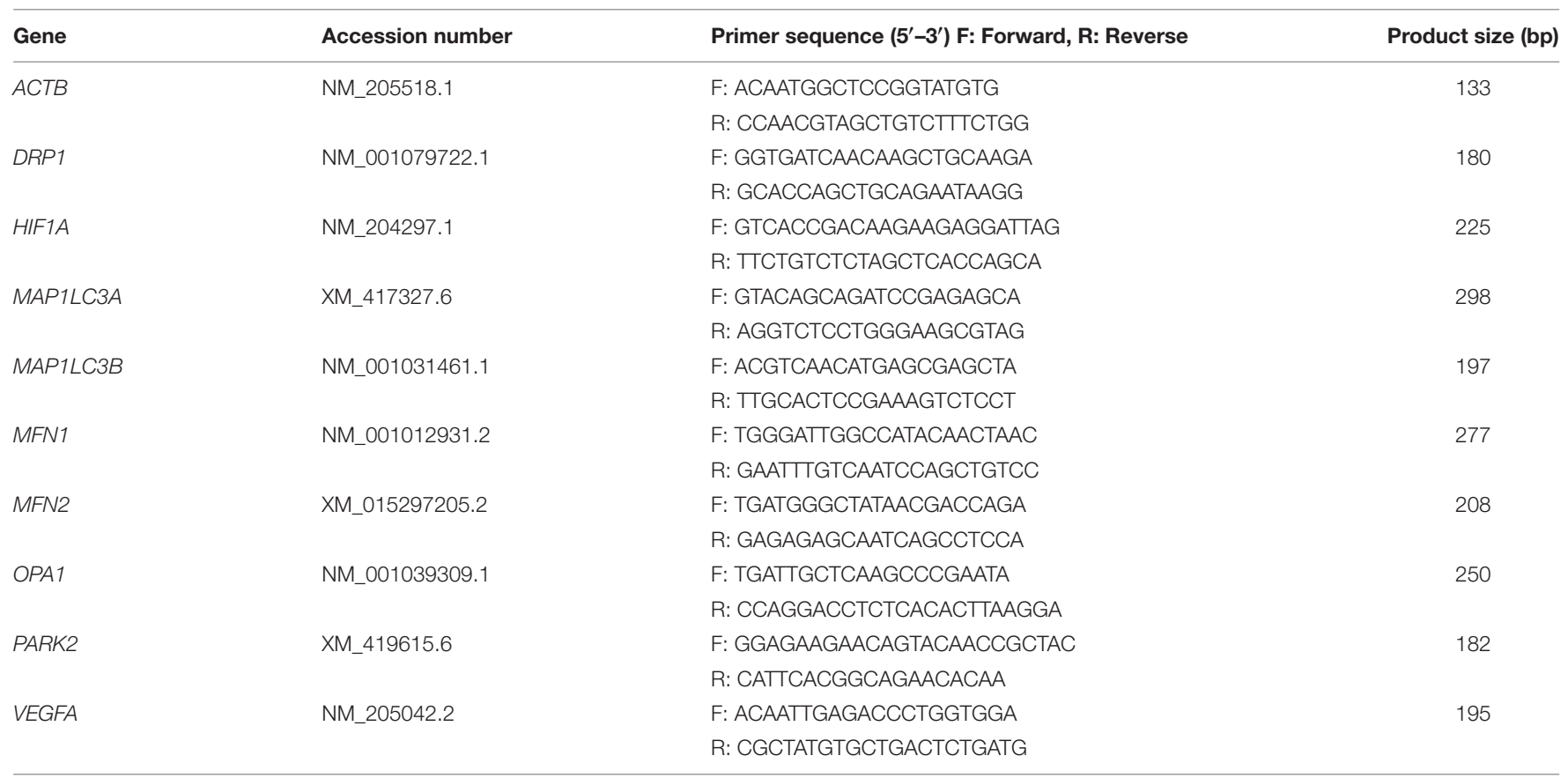

ACTB, actin, beta; DRP1, dynamin-related protein 1; HIF1A, hypoxia inducible factor 1 subunit alpha; MAP1LC3A, B, microtubule associated protein 1 light chain 3 alpha, beta; MFN1, 2, mitofusion 1, 2; OPA1, OPA1, mitochondrial dynamin like GTPase; PARK2, parkin RBR E3 ubiquitin protein ligase; VEGFA, vascular endothelial growth factor $A$.

of palpation and wing lift examination (Supplementary Table 1). Comparison and correlation analysis between PM histopathology and these clinical data were performed; however, no significant associations were detected (Supplementary Tables 2, 3).

\section{Ultrastructural Observation of PM Myofibers}

In healthy myofibers, both normal, small mitochondria containing clear intrastructures and swelled round mitochondria were localized within the intact myofibrils, which are composed of myosin (Figure 2A). In the myofibers with numerous small vacuoles, spherical structures with a single-membrane containing degraded mitochondria, reflecting autophagosome-like vacuoles, were observed (Figure 2B). The myofibrils around the vacuole clearly showed intact morphology. Myofiber regeneration by fusion of multiple myoblasts was also observed (Figure 2C). In the cytosol of the myoblasts, spherical structures with a double-membrane containing normal mitochondria and other organelles such as endoplasmic reticulum, reflecting typical autophagosomes, were observed (Figure 2C).

\section{Relationship Between Hypoxia and Histopathological Observations in the PM}

To examine the association of the muscle hypoxic conditions in WB with the histopathological observations such as fibrosis and hypertrophy in the myofibers, the correlations between the hypoxia-adaptive genes $H I F 1 A$ and vascular endothelial growth factor A (VEGFA), and the muscle histopathological indices FA and CM were analyzed (Table 3). Although the expression of HIF1A did not show significant correlations with either histopathological index, that of VEGFA was significantly negatively correlated with both pathological indices.

\section{Relationship Between Mitochondrial Dynamics and Pathological Observations, Hypoxia, or Autophagy/Mitophagy in the PM}

To reveal the association of the mitochondrial dynamics with the histopathological and hypoxic conditions in the PMs, the correlations between the expression of mitochondrial fusion[mitofusion 1, 2 (MFN1, MFN2) and mitochondrial dynaminlike GTPase (OPA1)] and fission [dynamin-related protein 1 (DRP1)]-related genes and the histopathological indices (FA and $\mathrm{CM}$ ) or the expression of hypoxia-adaptive genes HIF1A and VEGFA were analyzed (Table 4). The expression of MFN1 and MFN2 was significantly negatively correlated with CM and both FA and CM, respectively. Alternatively, DRP1 and HIF1A expression significantly positively correlated. In addition, the expression of all mitochondrial dynamics-related genes including MFN1, MFN2, OPA1, and DRP1 showed strong significantly positive correlation with that of VEGFA.

Based on the ultrastructural observations of the active metabolism of mitochondria as revealed by autophagylike vacuoles, the correlations between the expression of mitochondrial dynamics-related genes and that of autophagy/mitophagy-related genes were also analyzed (Table 4). Specifically, the microtubule-associated protein 1 light chain 


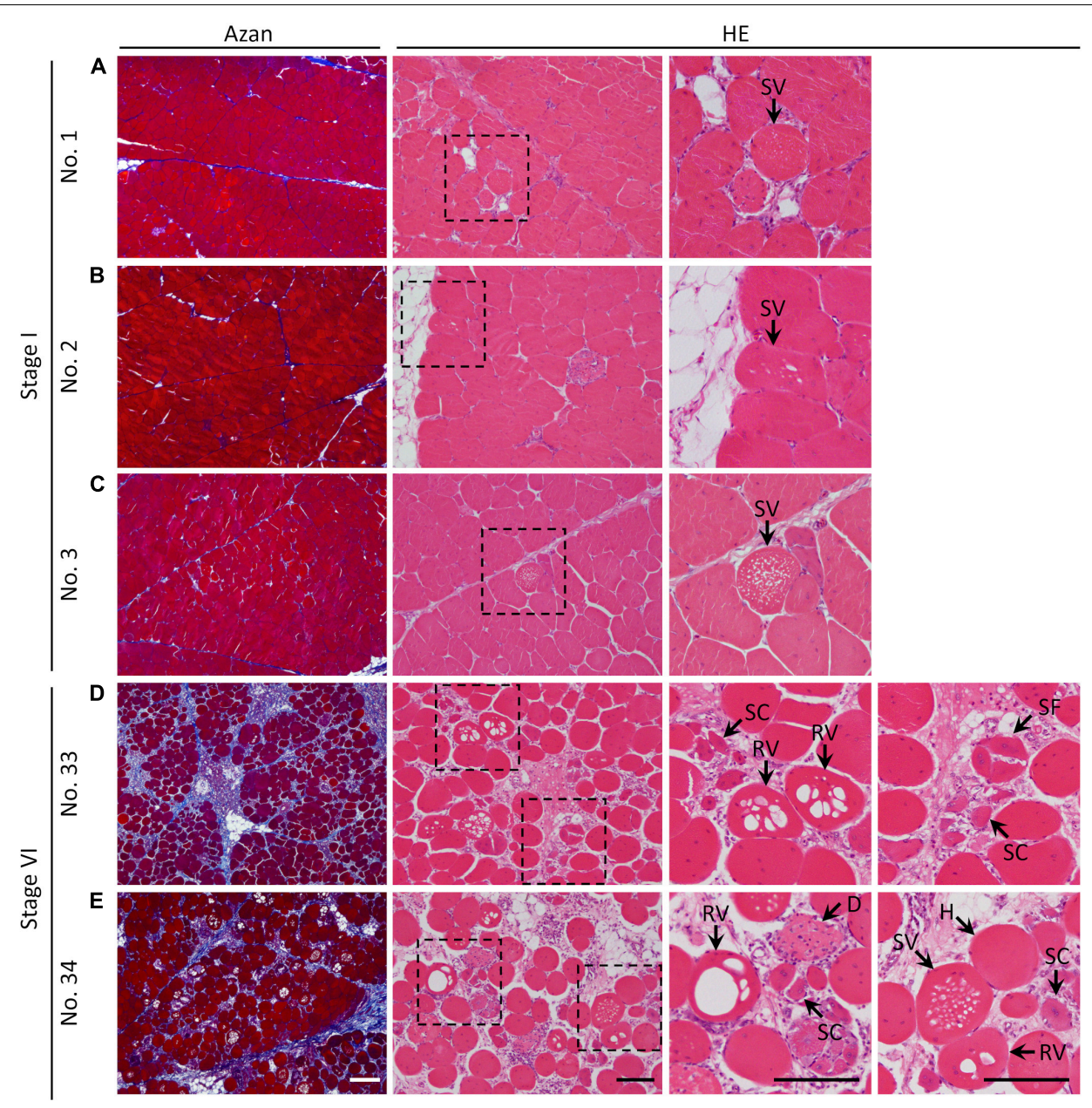

FIGURE 1 | Histology of the pectoralis major muscle of broilers stained with Azan and hematoxylin-eosin. (A-E) Histological observations of the pectoralis major muscle of (A) sample number 1 (stage I; FA, 7.24\% and CM, 0.70), (B) 2 (stage I; FA, 9.06\% and CM, 0.69), (C) 3 (stage I; FA, 13.25\% and CM, 0.68), (D) 33 (stage $\mathrm{VI}$; FA, 40.90\% and CM, 0.70), and (E) 34 (stage VI; FA, 40.97\% and CM, 0.76). The squares indicated by dashed lines are magnified on the right. White bar = 200 mm, black bar = 100 mm. SV, myofiber with numerous small vacuoles; RV, myofiber with large-rimmed vacuoles; H, hypertrophy of myofiber; SC, myofiber with small caliber; SF, split fiber; D, myofiber degeneration; FA, fibrotic area in the muscle; CM, circularity of myofibers.

3 alpha, beta $(M A P 1 L C 3 A, B)$ genes, also termed LC3A and $\mathrm{LC} 3 \mathrm{~B}$, constitute the primary genes related to autophagosome development and maturation (Schaaf et al., 2016), whereas parkin RBR E3 ubiquitin protein ligase (PARK2) acts as a specific regulator of mitophagy (Sato et al., 2018). We found that the expression of MFN1, MFN2, OPA1, and DRP1 was significantly positively correlated with that of all three autophagy-/mitophagy-related genes.

We further compared the expression levels of all examined genes among the six stages. No significant stage-related differences were observed in the expression of any of the examined genes (Figures 3A-C).

\section{DISCUSSION}

The histology of broiler PMs revealed myofiber hypertrophy and internal fibrosis, consistent with previous reports (Sihvo et al., 2014; Velleman and Clark, 2015), whereas the values of FA and CM histopathological indices differed among individuals, revealing that WB severity in the broilers varies during later life. Myofiber vacuolization was observed in all PMs analyzed in this study. Myofibers with numerous small vacuoles were apparent in the PMs with both mild and more severe FA and CM, whereas those with large-rimmed vacuoles frequently occurred in the PMs with more severe index scores. These vacuoles are thus termed autophagic vacuoles (AVs) in skeletal muscle (Malicdan and Nishino, 2012), and their appearance therein constitutes the pathognomonic morphological hallmark of autophagic vacuolar myopathy, which is a group of human hereditary myopathies including Pompe and Danon disease (Nishino, 2006; Cho and Noguchi, 2013; Castets et al., 2016). It has been reported that rimmed vacuoles are outcomes of impaired normal autophagy and represent frequent pathological characteristics in the chronic muscle damage of atrophy and myopathies (Suzuki et al., 2002; Sato et al., 2018). During autophagy, the accumulation of 
TABLE 2 | The fibrotic area (FA) in muscle, circularity of myofibers (CM), and stage classified by the FA in each bird.

\begin{tabular}{|c|c|c|c|c|c|}
\hline Sample No. & FA (\%) & CM & Stage & Mean \pm SE $(\%)$ of FA in each stage & Mean \pm SE of $\mathrm{CM}$ in each stage \\
\hline 1 & 7.24 & 0.70 & I & $13.45 \pm 1.81^{\| l, I I, I V, V, V I}$ & $0.67 \pm 0.02^{V}$ \\
\hline 2 & 9.06 & 0.69 & I & - & - \\
\hline 3 & 13.25 & 0.68 & I & - & - \\
\hline 4 & 16.31 & 0.67 & 1 & - & - \\
\hline 5 & 17.10 & 0.68 & 1 & - & - \\
\hline 6 & 17.76 & 0.57 & I & - & - \\
\hline 7 & 21.53 & 0.67 & $\|$ & $23.59 \pm 0.58^{\mathrm{IV}, \mathrm{V}, \mathrm{VI}}$ & $0.68 \pm 0.01$ \\
\hline 8 & 23.28 & 0.70 & $\|$ & - & - \\
\hline 9 & 24.06 & 0.67 & $\|$ & - & - \\
\hline 10 & 24.13 & 0.67 & $\|$ & - & - \\
\hline 11 & 24.92 & 0.68 & $\|$ & - & - \\
\hline 12 & 25.86 & 0.61 & III & $27.05 \pm 0.64^{\mathrm{IV}, \mathrm{V}, \mathrm{VI}}$ & $0.68 \pm 0.02$ \\
\hline 13 & 26.15 & 0.74 & III & - & - \\
\hline 14 & 26.19 & 0.66 & III & - & - \\
\hline 15 & 26.28 & 0.74 & III & - & - \\
\hline 16 & 27.95 & 0.70 & III & - & - \\
\hline 17 & 29.86 & 0.66 & III & - & - \\
\hline 18 & 31.14 & 0.73 & IV & $31.96 \pm 0.51^{\vee, \mathrm{Vl}}$ & $0.70 \pm 0.01$ \\
\hline 19 & 31.18 & 0.69 & IV & - & - \\
\hline 20 & 31.35 & 0.68 & IV & - & - \\
\hline 21 & 32.05 & 0.70 & IV & - & - \\
\hline 22 & 34.08 & 0.72 & IV & - & - \\
\hline 23 & 36.92 & 0.74 & V & $38.15 \pm 0.35$ & $0.74 \pm 0.01$ \\
\hline 24 & 37.00 & 0.74 & V & - & - \\
\hline 25 & 37.37 & 0.74 & V & - & - \\
\hline 26 & 37.80 & 0.75 & V & - & - \\
\hline 27 & 37.93 & 0.69 & V & - & - \\
\hline 28 & 38.05 & 0.75 & V & - & - \\
\hline 29 & 39.21 & 0.75 & V & - & - \\
\hline 30 & 39.47 & 0.74 & V & - & - \\
\hline 31 & 39.61 & 0.71 & V & - & - \\
\hline 32 & 40.65 & 0.70 & VI & $40.88 \pm 0.08$ & $0.72 \pm 0.01$ \\
\hline 33 & 40.90 & 0.70 & $\mathrm{VI}$ & - & - \\
\hline 34 & 40.97 & 0.76 & VI & - & - \\
\hline 35 & 41.00 & 0.72 & VI & - & - \\
\hline
\end{tabular}

Significant differences among stages are indicated by superscripted stage no. (I, II, III, IV, V, and VI) $(P<0.05$, Tukey test).

protein aggregates disturbs the maturation and fusion steps of autophagosome formation and enlarges the autophagic vesicles, where the rimmed vacuoles originate (Castets et al., 2016). Therefore, the rimmed vacuoles frequently observed in the PMs of broilers with more severe FA and CM implies the pathological condition of autophagy, indicating the close relationship between the altered autophagy and WB severity.

The ultrastructural observations of mitochondrial morphology confirmed that both physiological and pathological autophagies occur in the broiler skeletal muscles. Even in the histologically healthy myofibers, not only normal but also swelled and degenerated mitochondria are distributed within the myofibrils. In the myofibers exhibiting numerous small vacuoles, the AVs appeared to engulf the degenerated mitochondria. Moreover, because these AVs were enveloped by a single membrane, it was inferred that they were in the degradation stage during autophagy, in which the autophagosome double membrane is degraded by lysosomes as an autolysosome (Eskelinen et al., 2011). In mouse studies, deficiency of apolipoprotein B messenger RNA (mRNA) editing enzyme catalytic polypeptide 2 (APOBEC2), a member of the zincdependent cytidine deaminase protein family, has been reported to lead to a loss of skeletal muscle mass and atrophy (Sato et al., 2009). Notably, APOBEC2 deficiency does not affect the sarcomeric structure but rather causes mitochondrial morphological abnormalities in the murine skeletal myofibers, with the abnormal mitochondria being surrounded by AVs and removed by mitophagy (Sato et al., 2018). In comparison, in the myofibers of broilers analyzed in the present study, the myofibrils surrounding the AVs were ultrastructurally healthy and intact, indicating that the engulfment and digestion of these vacuoles is limited to the mitochondria likely through 

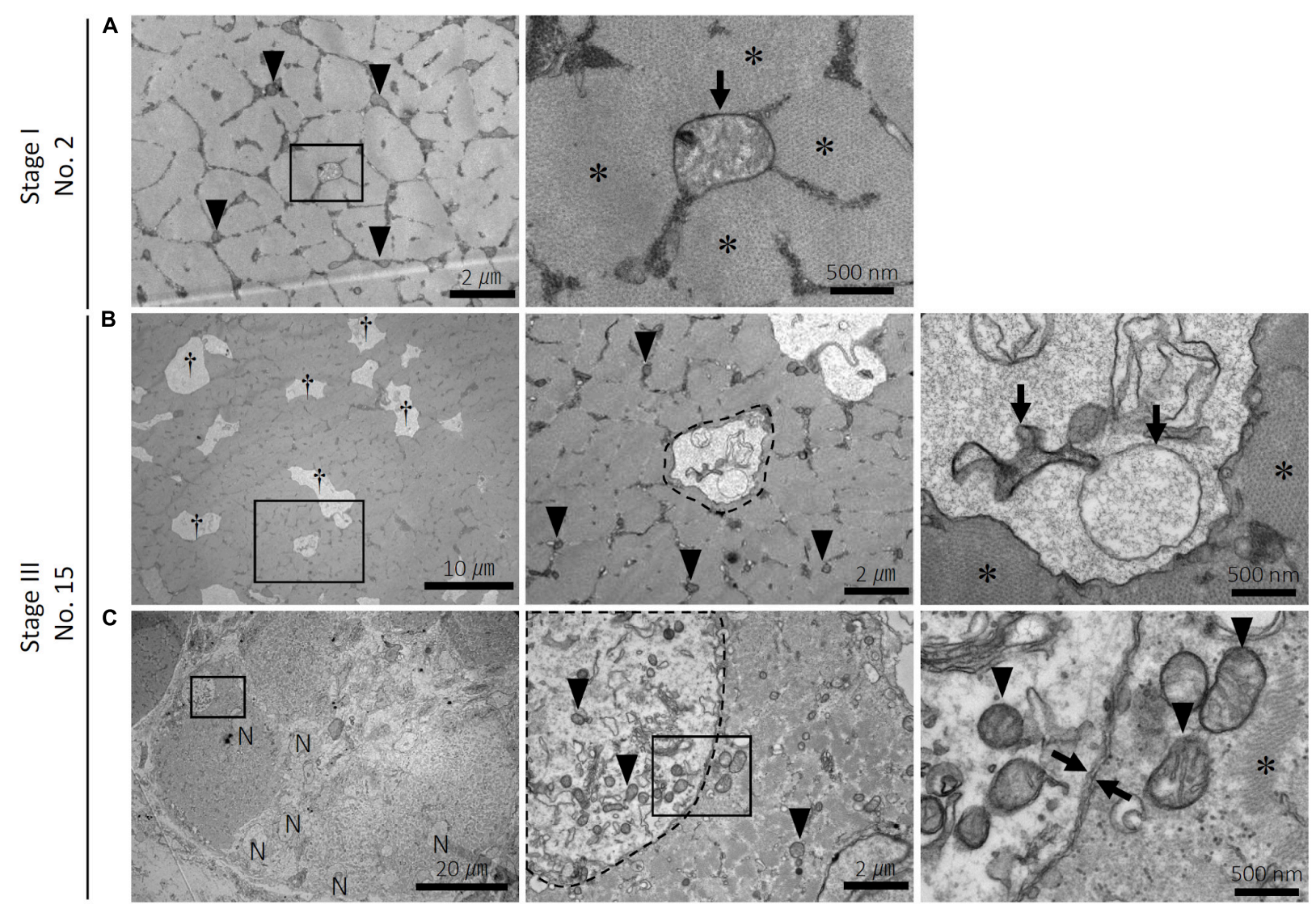

FIGURE 2 | Ultrastructure of the myofibers in the pectoralis major muscle of broilers. Ultrastructure of the pectoralis major muscle of (A) sample number 2 (stage I; FA, 9.06\% and CM, 0.69) and (B,C) 15 (stage III; FA, 26.28\% and CM, 0.74). FA, fibrotic area in the muscle; CM, circularity of myofibers. (A) Distribution of small and clear mitochondria (arrowheads) and a swelled mitochondrion (arrow) within myofibrils (asterisks) in the normal myofiber. The square indicated by black line is magnified on the right. (B) Numerous small vacuoles (daggers) are distributed within myofibrils (asterisks) in the myofibers. The vacuole (surrounded by a dashed line) envelops the swelled and degenerated mitochondria (arrows). The square indicated by black line is magnified on the right. Arrowheads: small and clear mitochondria. (C) Multiple myoblasts accumulated in the pectoralis major muscle. The autophagosome with a double membrane (between arrows) in the myoblast envelops organelles and the small and clear mitochondria (arrowheads). The squares indicated by black line are magnified on the right. N, nuclei in the myoblast; asterisks, myofibrils.

the mitophagy process. Specifically, to maintain skeletal muscle homeostasis, muscle repair and remodeling promotes the proliferation of satellite cells as myoblasts, which activates their myogenic differentiation (Sin et al., 2016). The autophagy and/or mitophagy in myoblasts are physiologically required in order to regenerate the skeletal muscle by myoblast differentiation (Sin et al., 2016; Tokutake et al., 2019). Herein, we observed typical autophagosomes with double membranes engulfing organelles in the myoblasts of the broiler PM. Therefore, we consider that, in the broilers, the autophagy/mitophagy that occurs via AVs in the myofibers and via autophagosomes in the myoblasts has different biological interpretations.

Hypoxia-inducible factorl is a heterodimer composed of an alpha and a beta subunit, which are respectively induced by hypoxia and constitutively expressed (Greijer and van der Wall, 2004). HIF $1 \alpha$ mediates the primary transcriptional response to hypoxia by binding to conserved sequences in the promoter regions of various hypoxia-adaptive genes (Majmundar et al., 2010; Thomas and Ashcroft, 2019). VEGF is the principal
TABLE 3 | Spearman's correlation coefficient ( $\rho$ ) between the hypoxia-adaptive gene expression and muscle histopathological indices.

\begin{tabular}{lccc}
\hline \multirow{2}{*}{ Gene } & \multicolumn{2}{c}{ Histopathological index } \\
\cline { 3 - 4 } & & FA & CM \\
\hline HIF1A $(n=35)$ & $\rho$ & 0.2616 & 0.2392 \\
VEGFA $(n=35)$ & $P$ & 0.129 & 0.1664 \\
& $\rho$ & -0.4476 & -0.584 \\
& $P$ & $0.007^{\star *}$ & $0.0002^{\star \star \star}$
\end{tabular}

HIF1A, hypoxia inducible factor 1 subunit alpha; VEGFA, vascular endothelial growth factor A; FA, fibrotic area in the muscle; CM, circularity of myofibers. ${ }^{* *} P<0.01,{ }^{* *} P<0.001$

transcriptional target of HIF1 $\alpha$ and induces angiogenesis in the hypoxic condition, which increases the oxygen supply (Majmundar et al., 2010). In skeletal muscles, the hypoxia consequent to exercise and ischemia is known to induce angiogenesis through HIF1-VEGF signaling (Ohno et al., 2012). 


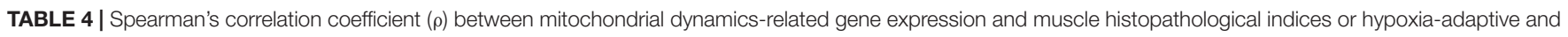
autophagy-/mitophagy-related gene expression.

\begin{tabular}{|c|c|c|c|c|c|c|c|c|}
\hline \multirow[t]{3}{*}{ Gene } & & \multicolumn{7}{|c|}{ Parameter } \\
\hline & & \multicolumn{2}{|c|}{ Histopathological index } & \multicolumn{2}{|c|}{ Hypoxia-adaptive gene expression } & \multicolumn{3}{|c|}{ Autophagy-/mitophagy-related gene expression } \\
\hline & & FA & $\mathrm{CM}$ & HIF1A & VEGFA & MAP1LC3A & MAP1LC3B & PARK2 \\
\hline MFN1 & $\rho$ & -0.279 & -0.3364 & 0.2501 & 0.6804 & 0.5882 & 0.5832 & 0.565 \\
\hline$(n=35)$ & $P$ & 0.1046 & $0.0482^{*}$ & 0.1473 & $<0.0001^{\star \star \star}$ & $0.0002^{\star \star \star}$ & $0.0002^{\star \star \star}$ & $0.0004^{\star \star \star}$ \\
\hline MFN2 & $\rho$ & -0.3734 & -0.4451 & 0.0482 & 0.7555 & 0.6384 & 0.6563 & 0.4866 \\
\hline$(n=35)$ & $P$ & $0.0271^{\star}$ & $0.0074^{\star \star}$ & 0.7834 & $<0.0001^{\star \star \star}$ & $<0.0001^{\star \star \star}$ & $<0.0001^{\star \star \star}$ & $0.003^{\star \star}$ \\
\hline OPA1 & $\rho$ & -0.1499 & -0.2574 & 0.2714 & 0.5644 & 0.6339 & 0.5933 & 0.3854 \\
\hline$(n=35)$ & $P$ & 0.3902 & 0.1355 & 0.1147 & $0.0004^{\star \star \star}$ & $<0.0001^{\star \star \star}$ & $0.0002^{\star \star \star}$ & $0.0222^{*}$ \\
\hline DRP1 & $\rho$ & -0.2734 & -0.237 & 0.3353 & 0.6204 & 0.688 & 0.6796 & 0.5232 \\
\hline$(n=35)$ & $P$ & 0.112 & 0.1705 & $0.049^{\star}$ & $<0.0001^{\star \star \star}$ & $<0.0001^{\star \star \star}$ & $<0.0001^{\star \star \star}$ & $0.0013^{\star *}$ \\
\hline
\end{tabular}

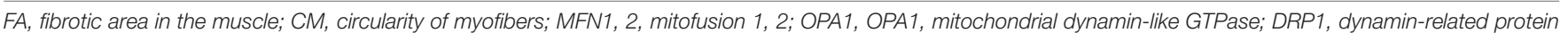

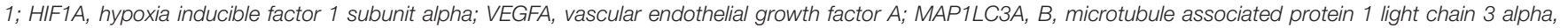
beta; PARK2, parkin RBR E3 ubiquitin protein ligase. ${ }^{\star} P<0.05,{ }^{\star *} P<0.01$, ${ }^{\star * *} P<0.001$.

A

HIF1A

VEGFA
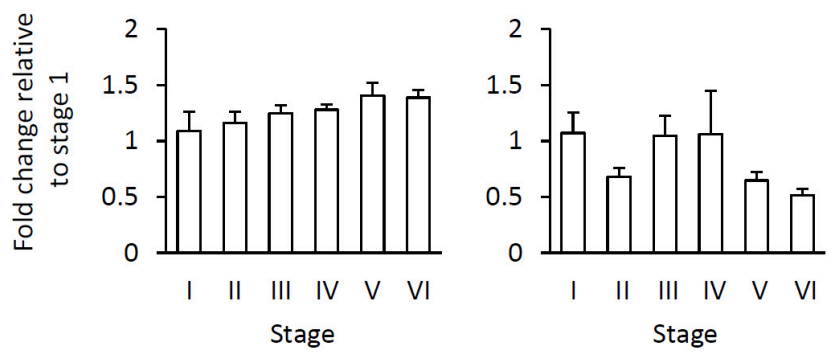

B

MFN1

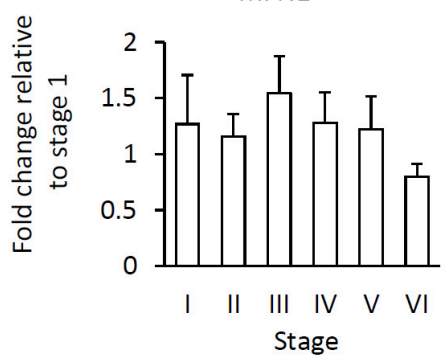

MFN2

OPA1

DRP1

C
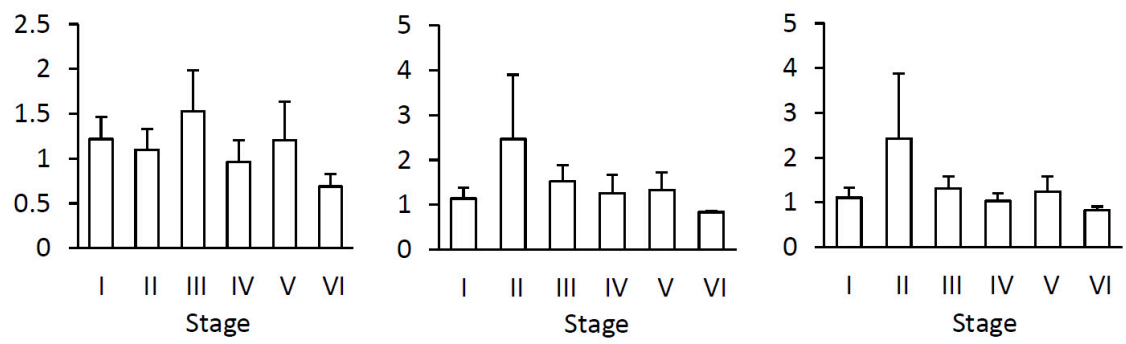

MAP1LC3B

PARK2
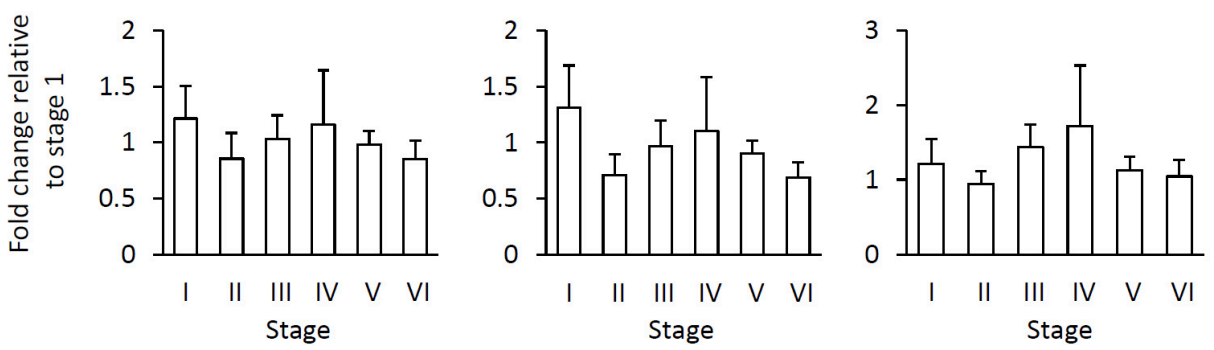

FIGURE 3 | Relationship of gene expression levels in the various stages to fibrotic area in the muscle. (A) Hypoxia-adaptive gene expression including hypoxia inducible factor 1 subunit alpha (HIF1A) and vascular endothelial growth factor A (VEGFA). (B) Mitochondrial dynamics-related gene expression including mitofusion 1, 2 (MFN1, 2), OPA1, mitochondrial dynamin-like GTPase (OPA1), and dynamin-related protein 1 (DRP1). (C) Autophagy-/mitophagy-related gene expression including microtubule-associated protein 1 light chain 3 alpha, beta (MAP1LC3A, B) and parkin RBR E3 ubiquitin protein ligase (PARK2). Data represent the means \pm SE [stage I $(n=6)$, stage II $(n=5)$, stage III $(n=6)$, stage IV $(n=5)$, stage $\vee(n=9)$, and stage VI $(n=4)$, Tukey test $(P<0.05)$ ]. 
In the PMs of the broilers, VEGFA expression was significantly negatively correlated with the muscle pathological indices FA and $\mathrm{CM}$, indicating that the decreased angiogenesis and oxygen supply in the skeletal muscle worsened the symptoms of WB. As hypoxia-mediated HIF1 induction occurs at the level of protein stability whereas HIF1A mRNA expression remains unchanged (Greijer and van der Wall, 2004), our correlation analysis results suggest that HIF1A expression and WB severity are not related in the broiler PMs.

Rather, the small caliber myofibers and split fibers observed in the PMs with more severe FA and CM, in which the angiogenesis is insufficient for supplying oxygen, are considered to be the outcomes of impaired myofiber regeneration consequent to the severe hypoxia. In general, the hypoxia-mediated impairment of skeletal muscle regeneration promotes the loss of muscle mass and atrophy (Neel et al., 2013; Romanello and Sandri, 2016). However, in broilers, the skeletal muscles develop fibrosis within the degenerated myofibers instead of decreasing their mass, resulting in the retention of high PM mass. In comparison, in the field of cancer medicine, it is recognized that following repeated and chronic periods of hypoxia, selection for resistance to the hypoxia-induced HIF1 response may occur, which exacerbates hypoxia-adaptive pathologies (Greijer and van der Wall, 2004). We consider that the chronic hypoxia in the broiler PMs might similarly alter the reactivity to HIF signaling and attenuate PM adaptability to the hypoxia.

Mitochondria undergo continuous cycles of fusion and fission, referred as mitochondrial dynamics, to maintain their physiological functions (Tilokani et al., 2018). Mitochondrial fusion, whereby one mitochondrion is produced from the union of two mitochondria, promotes the reduction and dilution of damage in each mitochondria by mixing their matrix contents for complementation (Byrne et al., 2019). MFN1, MFN2, and OPA1 encode key factors for mediating mitochondrial fusion (Hall et al., 2014). Alternatively, mitochondrial fission, whereby one mitochondrion is divided into two daughter mitochondria, not only regulates the amount and distribution of mitochondria but also plays a role in disposing their deteriorated contents by autophagy or mitophagy. Mitochondrial fission is regulated mainly by DRP1 expression (Balog et al., 2016; Byrne et al., 2019).

Mitochondrial quality control is necessary in highly structured skeletal muscles (Favaro et al., 2019). In the PMs of broilers,

PM of healthy broiler or mild WB
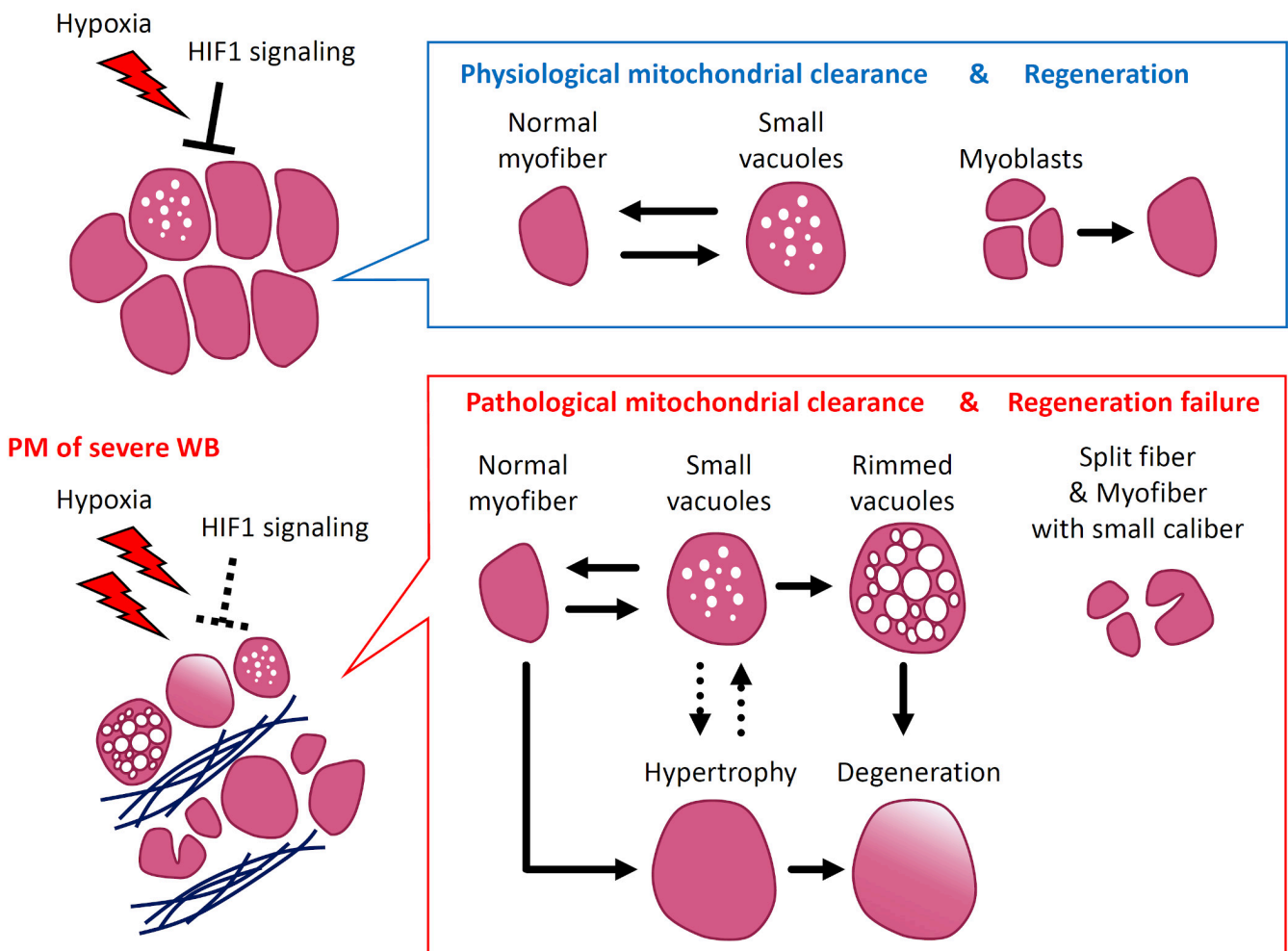

Pathological mitochondrial clearance \& Regeneration failure
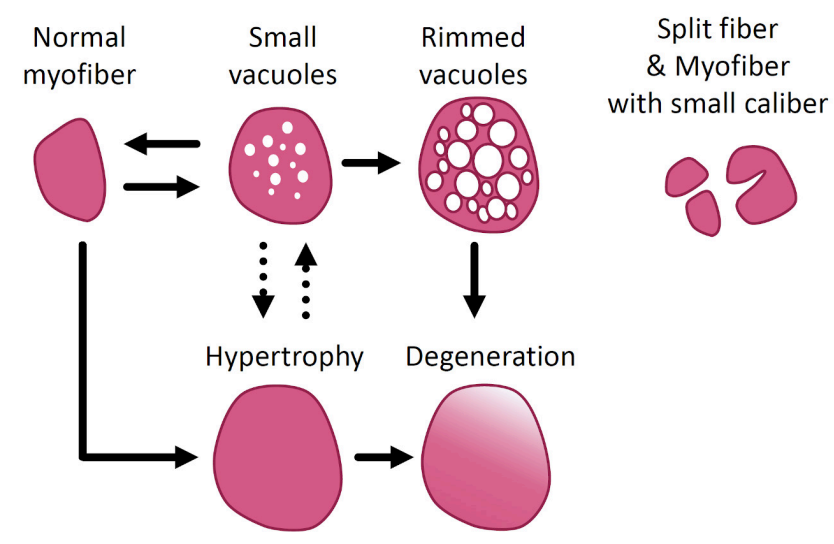

FIGURE 4 | Scheme of wooden breast syndrome (WB) pathogenesis focusing on mitochondrial clearance and myofiber pathology. In pectoralis major muscles (PM) with no or mild WB, the adaptation to hypoxia by HIF1 signaling induces physiological mitochondrial clearance mediated by mitochondrial fusion, fission, and autophagy/mitophagy, which corresponds with the appearance of myofibers with small vacuoles. At this stage, PM repair appears to occur through the differentiation of myoblasts into myofibers. Alternatively, in the muscles exhibiting severe WB, the acquired resistance to hypoxia alters the regulation of mitochondrial clearance. The accumulated damage in mitochondria exacerbates the various pathological characteristics such as myofibers with rimmed vacuoles, along with their hypertrophy and degeneration. Under severe hypoxia, the regeneration of myofibers fails, which corresponds with the frequent appearance of split fibers and myofibers with small caliber. HIF1, hypoxia-inducible factor 1. 
the expression of these mitochondrial dynamics-related genes showed strong positive correlation with that of VEGFA, suggesting that active and continuous mitochondrial dynamics affords the maintenance and clearance of mitochondria damaged by hypoxia at the early stage of $\mathrm{WB}$, during which active angiogenesis occurs. The negative correlation between mitochondrial dynamics-related genes with histopathological indices, especially that of MFN1 and MFN2 with FA and/or CM, indicated that WB pathology deteriorates in conjunction with reduced mitochondrial dynamics, especially fusion.

The series of mitochondrial fusion, fission, and mitophagy is integrated into the sequential mechanism of damaged mitochondria maintenance; i.e., the reorganization and segregation of damaged mitochondrial contents into daughter mitochondria that are eliminated by autophagy and/or mitophagy (Twig et al., 2008). In particular, the muscle specific loss of Drp1 in mouse resulted in myofiber death, atrophy, and degeneration of skeletal muscles, involving inhibition of autophagy and mitophagy (Favaro et al., 2019). The strong positive correlation between the expression of mitochondrial dynamics- and autophagy-/mitophagy-related genes observed in the present study suggested that the maintenance of mitochondrial health is highly controlled by this sequential mechanism in broiler skeletal muscles as well. Specifically, whereas the active maintenance of mitochondrial health by their fusion, fission, and autophagy/mitophagy is triggered in response to the hypoxia at the early stage of $\mathrm{WB}$, the acquired resistance to the HIF1 signaling under conditions of chronic hypoxia appears to lead to the attenuated regulation of mitochondrial maintenance by appropriate mitochondrial dynamics and autophagy/mitophagy. In addition, the broilers cannot voluntarily regulate energy balance and exhibit hyperphagia, resulting in excessive accumulation of energy (Richards, 2003). In the situation of decreased energy demand and increased supply, mitochondria progress through fission and fragmentation to adapt to the nutrient excess, which is associated with impaired mitochondrial function (Liesa and Shirihai, 2013). The damaged mitochondria in PMs mediated by excess nutrients may therefore also be screened out through the process of mitochondrial clearance in broilers.

In conclusion, our findings demonstrate that the PMs of broilers support the mechanism of physiological mitochondrial clearance, which is defined as the mechanism for maintaining mitochondria damaged by hypoxia as mediated by their continuous fusion, fission, and autophagy/mitophagy. In broilers in which the muscle is constantly exposed to hypoxia from early life owing to a genetic background that facilitates rapidly increasing PM mass, the HIF1-mediated response to hypoxia induces physiological mitochondrial clearance.

\section{REFERENCES}

Abasht, B., Mutryn, M. F., Michalek, R. D., and Lee, W. R. (2016). Oxidative stress and metabolic perturbations in wooden breast disorder in chickens. PLoS One 11:e0153750. doi: 10.1371/journal.pone.015 3750
However, the repeated and chronic hypoxia in the PMs weakens HIF1 signaling, exacerbating the oxygen deficiency and reducing physiological mitochondrial clearance. Eventually, the accumulation of damaged mitochondria would induce myofiber hypertrophy, degeneration, and reduced regeneration in the PMs along with fibrosis, reflecting the pathological characteristics of severe WB (Figure 4). This study revealed the essential role of physiological and pathological mitochondrial clearance in skeletal muscle in the pathogenesis of severe WB. Thus, our findings suggest that suppressing hypoxic stress from the initial stage may be crucial to prevent the development of severe WB.

\section{DATA AVAILABILITY STATEMENT}

The raw data supporting the conclusions of this article will be made available by the authors, without undue reservation, to any qualified researcher.

\section{ETHICS STATEMENT}

The animal study was reviewed and approved by the Rakuno Gakuen University Institutional Animal Care and Use Committee.

\section{AUTHOR CONTRIBUTIONS}

MarH, YH, YW, and MakH acquired the data. MarH, YW, and $\mathrm{MakH}$ analyzed the data. MarH, TI, and TW interpreted the results. TK, TT, TI, and TW designed the study. NT and HU provided technical assistance with transmission electron microscopy. MarH and TW wrote the first draft of the manuscript. All authors approved the final version of the manuscript.

\section{FUNDING}

This work was supported by Grant-in-Aid Scientific Research (C) from Japan Society for the Promotion of Science (\#18K05941, TW and \#17K08067, TI).

\section{SUPPLEMENTARY MATERIAL}

The Supplementary Material for this article can be found online at: https://www.frontiersin.org/articles/10.3389/fphys. 2020.00579/full\#supplementary-material

Bailey, R. A., Watson, K. A., Bilgili, S. F., and Avendano, S. (2015). The genetic basis of pectoralis major myopathies in modern broiler chicken lines. Poult. Sci. 94, 2870-2879. doi: 10.3382/ps/pev304

Balog, J., Mehta, S. L., and Vemuganti, R. (2016). Mitochondrial fission and fusion in secondary brain damage after CNS insults. J. Cereb. Blood Flow Metab. 36, 2022-2033. doi: 10.1177/0271678X16671528 
Byrne, J. J., Soh, M. S., Chandhok, G., Vijayaraghavan, T., Teoh, J. S., Crawford, S., et al. (2019). Disruption of mitochondrial dynamics affects behaviour and lifespan in Caenorhabditis elegans. Cell. Mol. Life Sci. 76, 1967-1985. doi: 10. 1007/s00018-019-03024-5

Castets, P., Frank, S., Sinnreich, M., and Rüegg, M. A. (2016). "Get the balance right": pathological significance of autophagy perturbation in neuromuscular disorders. J. Neuromuscul. Dis. 3, 127-155. doi: 10.3233/JND-16 0153

Cho, A., and Noguchi, S. (2013). "Autophagy in GNE myopathy," in Autophagy - A Double-Edged Sword - Cell Survival or Death?, ed. Y. Bailey (London: InTech), doi: $10.5772 / 55223$

Clark, D. L., and Velleman, S. G. (2016). Spatial influence on breast muscle morphological structure, myofiber size, and gene expression associated with the wooden breast myopathy in broilers. Poult. Sci. 95, 2930-2945. doi: 10.3382/ps/ pew243

Eskelinen, E. L., Reggiori, F., Baba, M., Kovács, A. L., and Seglen, P. O. (2011). Seeing is believing: the impact of electron microscopy on autophagy research. Autophagy 7, 935-956. doi: 10.4161/auto.7.9.15760

Favaro, G., Romanello, V., Varanita, T., Andrea Desbats, M., Morbidoni, V., Tezze, C., et al. (2019). DRP1-mediated mitochondrial shape controls calcium homeostasis and muscle mass. Nat. Commun. 10:2576. doi: 10.1038/s41467019-10226-9

Greijer, A. E., and van der Wall, E. (2004). The role of hypoxia inducible factor 1 (HIF-1) in hypoxia induced apoptosis. J. Clin. Pathol. 57, 1009-1014. doi: 10.1136/jcp.2003.015032

Griffin, J. R., Moraes, L., Wick, M., and Lilburn, M. S. (2018). Onset of white striping and progression into wooden breast as defined by myopathic changes underlying pectoralis major growth. Estimation of growth parameters as predictors for stage of myopathy progression. Avian Pathol. 47, 2-13. doi: 10.1080/03079457.2017.1356908

Hall, A. R., Burke, N., Dongworth, R. K., and Hausenloy, D. J. (2014). Mitochondrial fusion and fission proteins: novel therapeutic targets for combating cardiovascular disease. Br. J. Pharmacol. 171, 1890-1906. doi: 10. 1111/bph.12516

Hasegawa, Y., Hara, T., Kawasaki, T., Yamada, M., Watanabe, T., and Iwasaki, T. (2020). Effect of wooden breast on postmortem changes in chicken meat. Food Chem. 315:126285. doi: 10.1016/j.foodchem.2020.126285

Kawasaki, T., Iwasaki, T., Yamada, M., Yoshida, T., and Watanabe, T. (2018). Rapid growth rate results in remarkably hardened breast in broilers during the middle stage of rearing: a biochemical and histopathological study. PLoS One 13:e0193307. doi: 10.1371/journal.pone.0193307

Kawasaki, T., Yoshida, T., and Watanabe, T. (2016). Simple method for screening the affected birds with remarkably hardened pectoralis major muscles among broiler chickens. J. Poult. Sci. 53, 291-297. doi: 10.2141/jpsa.016 0036

Kuttappan, V. A., Huff, G. R., Huff, W. E., Hargis, B. M., Apple, J. K., Coon, C., et al. (2013). Comparison of hematologic and serologic profiles of broiler birds with normal and severe degrees of white striping in breast fillets. Poult. Sci. 92, 339-345. doi: 10.3382/ps.2012-02647

Liesa, M., and Shirihai, O. S. (2013). Mitochondrial dynamics in the regulation of nutrient utilization and energy expenditure. Cell Metab. 17, 491-506. doi: 10.1016/j.cmet.2013.03.002

Majmundar, A. J., Wong, W. J., and Simon, M. C. (2010). Hypoxia-inducible factors and the response to hypoxic stress. Mol. Cell 40, 294-309. doi: 10.1016/j.molcel. 2010.09.022

Malicdan, M. C. V., and Nishino, I. (2012). Autophagy in lysosomal myopathies. Brain Pathol. 22, 82-88. doi: 10.1111/j.1750-3639.2011. 00543.x

Malila, Y., Thanatsang, K., Arayamethakorn, S., Uengwetwanit, T., Srimarut, Y., Petracci, M., et al. (2019). Absolute expressions of hypoxia-inducible factor-1 alpha (HIF1A) transcript and the associated genes in chicken skeletal muscle with white striping and wooden breast myopathies. PLoS One 14:e0220904. doi: 10.1371/journal.pone.0220904

Mutryn, M. F., Brannick, E. M., Fu, W., Lee, W. R., and Abasht, B. (2015). Characterization of a novel chicken muscle disorder through differential gene expression and pathway analysis using RNA-sequencing. BMC Genomics 16:399. doi: 10.1186/s12864-015-1623-0
Neel, B. A., Lin, Y., and Pessin, J. E. (2013). Skeletal muscle autophagy: a new metabolic regulator. Trends Endocrinol. Metab. 24, 635-643. doi: 10.1016/j.tem. 2013.09.004

Nishino, I. (2006). Autophagic vacuolar myopathy. Semin. Pediatr. Neurol. 13, 90-95. doi: 10.1016/j.spen.2006.06.004

Ohno, H., Shirato, K., Sakurai, T., Ogasawara, J., Sumitani, Y., Sato, S., et al. (2012). Effect of exercise on HIF-1 and VEGF signaling. J. Phys. Fit. Sport. Med. 1, 5-16. doi: 10.7600/jpfsm.1.5

Papah, M. B., Brannick, E. M., Schmidt, C. J., and Abasht, B. (2018). Gene expression profiling of the early pathogenesis of wooden breast disease in commercial broiler chickens using RNA-sequencing. PLoS One 13:e0207346. doi: 10.1371/journal.pone.0207346

Petracci, M., Mudalal, S., Soglia, F., and Cavani, C. (2015). Meat quality in fastgrowing broiler chickens. Worlds Poult. Sci. J. 71, 363-374. doi: 10.1017/ S0043933915000367

Petracci, M., Soglia, F., Madruga, M., Carvalho, L., Ida, E., and Estévez, M. (2019). Wooden-breast, white striping, and spaghetti meat: causes, consequences and consumer perception of emerging broiler meat abnormalities. Compr. Rev. Food Sci. 18, 565-583. doi: 10.1111/1541-4337. 12431

Radaelli, G., Piccirillo, A., Birolo, M., Bertotto, D., Gratta, F., Ballarin, C., et al. (2017). Effect of age on the occurrence of muscle fiber degeneration associated with myopathies in broiler chickens submitted to feed restriction. Poult. Sci. 96, 309-319. doi: 10.3382/ps/pew270

Richards, M. P. (2003). Genetic regulation of feed intake and energy balance in poultry. Poult. Sci. 82, 907-916. doi: 10.1093/ps/82. 6.907

Romanello, V., and Sandri, M. (2016). Mitochondrial quality control and muscle mass maintenance. Front. Physiol. 6:422. doi: 10.3389/fphys.2015. 00422

Sato, Y., Ohtsubo, H., Nihei, N., Kaneko, T., Sato, Y., Adachi, S., et al. (2018). Apobec2 deficiency causes mitochondrial defects and mitophagy in skeletal muscle. FASEB J. 32, 1428-1439. doi: 10.1096/fj.20170 0493R

Sato, Y., Shimizu, M., Mizunoya, W., Wariishi, H., Tatsumi, R., Buchman, V. L., et al. (2009). Differential expression of sarcoplasmic and myofibrillar proteins of rat soleus muscle during denervation atrophy. Biosci. Biotechnol. Biochem. 73, 1748-1756. doi: 10.1271/bbb.90085

Schaaf, M. B. E., Keulers, T. G., Vooijs, M. A., and Rouschop, K. M. A. (2016). LC3/GABARAP family proteins: autophagy-(un)related functions. FASEB J. 30, 3961-3978. doi: 10.1096/fj.201600698R

Sihvo, H.-K., Immonen, K., and Puolanne, E. (2014). Myodegeneration with fibrosis and regeneration in the pectoralis major muscle of broilers. Vet. Pathol. 51, 619-623. doi: 10.1177/0300985813497488

Sin, J., Andres, A. M., Taylo, R., Weston, T., Hiraumi, Y., Stotland, A., et al. (2016). Mitophagy is required for mitochondrial biogenesis and myogenic differentiation of C2C12 myoblasts. Autophagy 12, 369-380. doi: 10.1080/ 15548627.2015.1115172

Suzuki, T., Nakagawa, M., Yoshikawa, A., Sasagawa, N., Yoshimori, T., Ohsumi, Y., et al. (2002). The first molecular evidence that autophagy relates rimmed vacuole formation in chloroquine myopathy. J. Biochem. 131, 647-651. doi: 10.1093/oxfordjournals.jbchem.a003147

Thai, T. Q., Nguyen, H. B., Saitoh, S., Wu, B., Saitoh, Y., Shimo, S., et al. (2016). Rapid specimen preparation to improve the throughput of electron microscopic volume imaging for three-dimensional analyses of subcellular ultrastructures with serial block-face scanning electron microscopy. Med. Mol. Morphol. 49, 154-162. doi: 10.1007/s00795-016-0134-7

Thomas, L. W., and Ashcroft, M. (2019). Exploring the molecular interface between hypoxia-inducible factor signalling and mitochondria. Cell. Mol. Life Sci. 76, 1759-1777. doi: 10.1007/s00018-019-03039-y

Tilokani, L., Nagashima, S., Paupe, V., and Prudent, J. (2018). Mitochondrial dynamics: overview of molecular mechanisms. Essays Biochem. 62, 341-360. doi: 10.1042/EBC20170104

Tokutake, Y., Yamada, K., Hayashi, S., Arai, W., Watanabe, T., and Yonekura, S. (2019). IRE1-XBP1 pathway of the unfolded protein response is required during early differentiation of C2C12 myoblasts. Int. J. Mol. Sci. 21:E182. doi: $10.3390 /$ ijms 21010182 
Twig, G., Hyde, B., and Shirihai, O. S. (2008). Mitochondrial fusion, fission and autophagy as a quality control axis: the bioenergetic view. Biochim. Biophys. Acta 1777, 1092-1097. doi: 10.1016/j.bbabio.2008. 05.001

Velleman, S. G., and Clark, D. L. (2015). Histopathologic and myogenic gene expression changes associated with wooden breast in broiler breast muscles. Avian Dis. 59, 410-418. doi: 10.1637/11097-042015Reg.1

Ziello, J. E., Jovin, I. S., and Huang, Y. (2007). Hypoxia-inducible factor (HIF)-1 regulatory pathway and its potential for therapeutic intervention in malignancy and ischemia. Yale J. Biol. Med. 80, 51-60.
Conflict of Interest: The authors declare that the research was conducted in the absence of any commercial or financial relationships that could be construed as a potential conflict of interest.

Copyright (c) 2020 Hosotani, Kawasaki, Hasegawa, Wakasa, Hoshino, Takahashi, Ueda, Takaya, Iwasaki and Watanabe. This is an open-access article distributed under the terms of the Creative Commons Attribution License (CC BY). The use, distribution or reproduction in other forums is permitted, provided the original author(s) and the copyright owner(s) are credited and that the original publication in this journal is cited, in accordance with accepted academic practice. No use, distribution or reproduction is permitted which does not comply with these terms. 